\title{
Resonance Induced by Repulsive Links
}

Teresa Vaz Martins and Raúl Toral

\begin{abstract}
In nonlinear systems, the right amount of noise can amplify the response to a weak periodic signal, by a phenomenon known as stochastic resonance ${ }^{1}$. It was shown that the same constructive role can be played by any source of disorder ${ }^{2}$ We study an Ising model in a network with disorder induced by the presence of both attractive and repulsive links. The system is subjected to a sub-threshold periodic signal, and the goal is to see how the response is enhanced for a given fraction of repulsive links. This can model a network of spinlike neurons with excitatory and inhibitory couplings. By means of numerical simulations, we find that there is an optimal probability of repulsive links, such that the coherent response is maximal, and we propose a mechanism to explain this resonance.
\end{abstract}

\section{Model}

We start with a spinlike network, where each node has two possible states: $\mu_{i}= \pm 1$ These states are assigned randomly at the beginning, and they can represent for instance the firing state of a neuron, or two different opinions

At each time step, a node is selected at random, and then the dynamics evolves with the consecutive application of the following steps:

- the selected node $i$ adopts the majority state of its neighbors. If the weight is zero, it doesn't change

Teresa Vaz Martins

Centro de Física do Porto (FCUP), 4169-007 Porto, Portugal, FCT, SFRH/BD/23709/2005 e-mail: tvmartins@fc.up.pt

Raúl Toral

IFISC, Instituto de Fisica Interdisciplinar y Sistemas Complejos (CSIC-UIB), E-07122 Palma de Mallorca, Spain, e-mail: raul@ifisc.uib.es 


$$
\mu_{i}=\operatorname{sign}\left(\sum \omega_{i j} \mu_{j}\right)
$$

with the relationship matrix

$$
\omega_{i j}=\left\{\begin{array}{c}
1, \operatorname{prob}(1-p) \\
-k, \text { prob } p
\end{array}\right.
$$

- with probability $|\operatorname{asin}(\Omega t)|$, it follows the external signal

$$
\mu_{i}=\operatorname{sign}(\sin (\Omega t)), \text { with prob }|\operatorname{asin}(\Omega t)|
$$

where $\Omega$ is the frequency of the external signal, and $a$ is its amplitude

After that, time increases by $1 / N$. Our results concern random networks, the system size is $\mathrm{N}=100$, and the average number of neighbors is 10 . Having tried other sizes, regular networks, or topologies such as a replicated random or a square network, we found the same type of phenomenon. The averages are over 1000 runs.

Together with the results for quenched disorder, sometimes we will also show what happens in a temporarily annealed version, where disorder is not correlated in time. The neighborhood is fixed but, for a transient time, the relationship matrix $w_{i j}$ is redefined randomly at each time step. This scenario can model a society where a diffusive animosity finally crystallizes into entrenched positions of friends and enemies, or in another context, the known fact that brain plasticity is higher in initial stages.

\section{Results}

As a measure of coherent response, we chose the spectral amplification factor $R$, defined as the ratio of the output power at the corresponding driving frequency to the input power.

The system becomes monostable, as an increasing probability of repulsive links degrades order (Fig 1). We found that the response to the subthreshold signal is maximal, for an intermediate proportion of repulsive links, within the transition region (Fig 2).

Fig. 1 Total Average Magnetization as a function of the probability of repulsive links

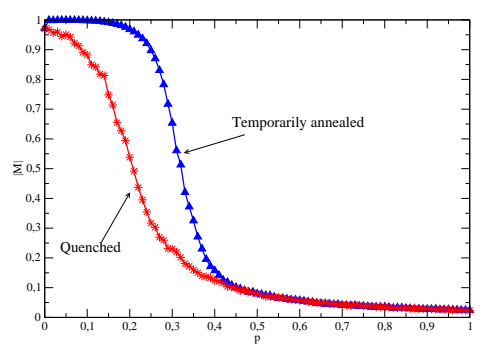


Fig. 2 Spectral amplification factor $R$ as a function of the probability of repulsive links, showing a peak at the right proportion of repulsive links

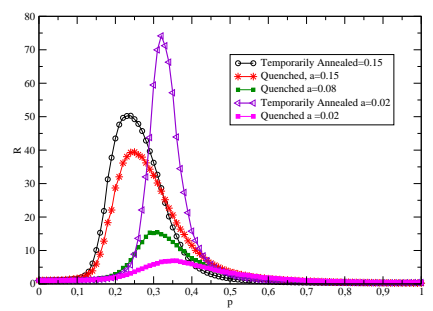

The amplitude of oscillations is amplified if we add the right proportion of repulsive links. The system oscillates with the signal's frequency.(Fig 3)

Fig. 3 Evolution of the total average magnetization in time, showing how the amplitude is greater at the optimal probability $p=0.26$. Here we show a quenched disorder case, but the temporarily annealed looks the same at this signal's intensity

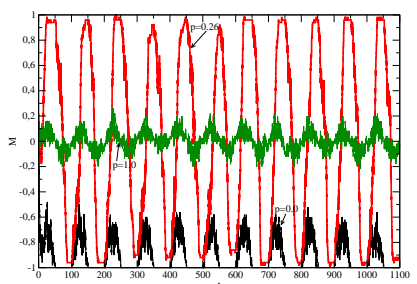

When the signal is weak, there is still an optimal probability, but the oscillations amplitude is less magnified.(Fig 4)

Fig. 4 Evolution of the total average magnetization with time, showing the increase in the amplitude of oscillations at the optimal probability $p=0.29$. Quenched disorder version. $a=0.08$.

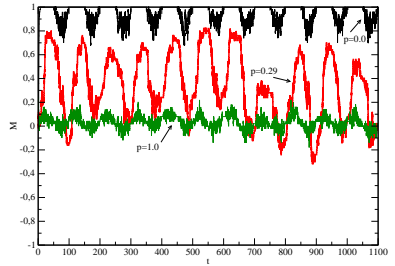

In the temporarily annealed disorder system, the signal's weakness is revealed in the coexistence of big and smaller amplitude of oscillations at the optimal probability, and also in a degradation of periodicity.(Fig 5)

Fig. 5 Evolution of the total average magnetization with time. Temporarily annealed disorder version. $a=0.02$.

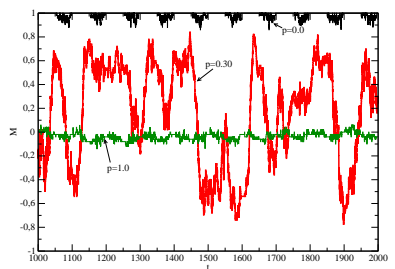


In stochastic resonance, with its prototypical bistable potential, we can speak of intra and inter-well oscillations ${ }^{3}$. To see if a similar picture applied, we have looked into how the stable states values were distributed. In the quenched disorder scenario they go from being concentrated at $M=-1$ or $M=1$, at $p=0$, to span a continuous wide range, at the optimal probability.

Fig. 6 Quenched disorder version: The optimal region is a region of many stable states, through which the system can progress in multi-steps.

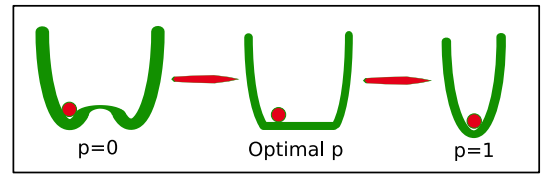

In the temporarily annealed version, at the optimal probability they are still concentrated at symmetric values, that get closer as the proportion of repulsive links increases. This system is more prone to respond in switch-like fashion.

Fig. 7 Temporarily annealed disorder version: Disorder lowers the potential barrier, and moves the wells closer

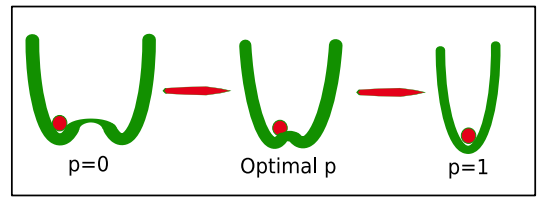

Meanwhile, the microscopic mechanism is the same. After a node has changed, it has two requirements, that explain where the optimal probability is located: it can not be forced to return to its previous state ( the effective local magnetization around a node should be close to zero). And it should spread its perturbation: (it is better to have attractive connections).

\section{Conclusions}

Competition can enhance a system response to an external signal. The model and rules are generic enough as to predict an application in several systems. Ways to optimize the mechanism: changing the network topology, play with parameters...

\section{References}

1. C. Tessone, C.R. Mirasso, R. Toral, J.D. Gunton: Diversity Induced Resonance, Phys. Rev. Lett. 97, 194101 (2006)

2. L. Gammaitoni, P. Hnggi, P. Jung and F. Marchesoni. Rev. Mod. Phys. 70, 225 (1998)

3. L. Alfonsi et al, Intrawell stochastic resonance versus interwell stochastic resonance in underdamped bistable systems, Phys. Rev. E 62, 299 - 302 (2000) 